Ciencias Agrarias / Agricultural Sciences

Cienc Tecn UTEQ (2020) 13(2) p 39-45 ISSN 1390-4051; e-ISSN 1390-4043

\title{
INÓCULO MICROBIANO CON CAPACIDAD CELULOLÍTICA PARA LA PRODUCCIÓN DE COMPOST EN MANABÍ-ECUADOR
}

\section{MICROBIAL INOCULUM WITH CELLULOLYTIC CAPACITY FOR COMPOST PRODUCTION IN MANABÍ-ECUADOR}

\author{
Ángel Monserrate Guzmán Cedeño1,2, Diego Efrén Zambrano-Pazmiño1, María Auxiliadora Conforme Álava1, Beatriz Rocío \\ Vera Vera1
}

1Escuela Superior Politécnica Agropecuaria de Manabi "Manuel Félix López", 10 de agosto No82 y Granada Centeno. Calceta, Manabi,Ecuador,aguzman@espam.edu.ec 2 Universidad Laica "Eloy Alfaro” de Manabi. Ciudadela universitaria vía San Mateo. Manta, Manabí, Ecuador.

Rec.: 19.08.2019. Acept.: 02.10.2020. Publicado el 30 de diciembre de 2020

\section{Resumen}

$\mathrm{D}$ os aislados de Trichoderma (T. harzianum, y T. longibrachiatium) y una cepa de Bacillus spp se probaron como inoculantes en el proceso de compostaje. Las pilas (cilindros de $0.33 \mathrm{~m}^{3}$ ) estuvieron conformadas por una mezcla de cáscara de maní y porquinaza, residuo de camas profundas de un hato porcino. A las pilas se aplicó diferentes tamaños de inóculo: $200 \mathrm{~mL} / \mathrm{m}^{3}$ (T1), $300 \mathrm{~mL} / \mathrm{m} 3$ (T2) $400 \mathrm{~mL} / \mathrm{m} 3$ (T3) y $0 \mathrm{~mL} / \mathrm{m}^{3}$ (testigo-T4). Durante el compostaje se controló la humedad (alrededor del 60\%) y temperatura que alcanzó $48.7^{\circ} \mathrm{C}$ en la etapa termofílica. A los 120 días del compostaje el pH osciló entre 6.94 y 7.12, los valores de conductividad eléctrica estuvieron entre 655 $1362 \mu \mathrm{S} \mathrm{cm}^{-1}$, la relación $\mathrm{C} / \mathrm{N}$ fue entre 16 y $29 \%$, el primer valor correspondió al tratamiento con mayor aportación de inóculo. La composición química del compost fue muy similar en los cuatro tratamientos, con ligeros incrementos donde se aplicó mayor cantidad de inóculo, lo que indica un estado más avanzado de transformación de la materia orgánica compostada. El tratamiento 3 presentó el menor contenido de coliformes totales y fecales al final del período de compostaje con 5 x $10^{1}$ y $1,33 \times 10^{1} \mathrm{NMP} / \mathrm{g}$ respectivamente. Se concluye que el inóculo contribuyó favorablemente al proceso de compostaje y por ende a la madurez y estabilidad del compost.

Palabras clave: carga microbiana, compostaje, actividades enzimáticas

\begin{abstract}
$\mathrm{T}$ wo Trichoderma isolates (T. harzianum, and $T$. longibrachiatium) and a Bacillus spp strain were tested as inoculants in the composting process. The piles (cylinders of $0.33 \mathrm{~m} 3$ ) were made up of a mixture of peanut shells and pig manure, residue from deep beds of a pig herd. Different sizes of inoculum were applied to the piles: $200 \mathrm{~mL} / \mathrm{m} 3$ (T1), $300 \mathrm{~mL} / \mathrm{m} 3$ (T2), 400 $\mathrm{mL} / \mathrm{m} 3$ (T3) and $0 \mathrm{~mL} / \mathrm{m} 3$ (control-T4). During composting, humidity (around 60\%) and temperature were controlled, reaching $48.7{ }^{\circ} \mathrm{C}$ in the thermophilic stage. 120 days after composting, the $\mathrm{pH}$ ranged between 6.94 and 7.12, the electrical conductivity values were between $655-1362 \mu \mathrm{S} \mathrm{cm}-1$, the $\mathrm{C} / \mathrm{N}$ ratio was between 16 and $29 \%$, the first value corresponded to the treatment with the highest contribution of inoculum. The chemical composition of the compost was very similar in the four treatments, with slight increases where a greater amount of inoculum was applied, which indicates a more advanced stage of transformation of the composted organic matter. Treatment 3 showed the lowest content of total and fecal coliforms at the end of the composting period with $5 \times 101$ and $1.33 \times 101$ MPN / $g$ respectively. It is concluded that the inoculum contributed favorably to the composting process and therefore to the maturity and stability of the compost.
\end{abstract}

Keywords: Microbial load, composting, enzymatic activities. 


\section{Introducción}

$\mathrm{L}$ a cantidad de residuos generados por la actividad agropecuaria se ha incrementado con el aumento de la demanda de alimentos, por el crecimiento de la población (Sabiiti, 2011), estos residuos se convierten en una fuente de contaminación para el ambiente y la salud pública cuando no se le proporciona un buen tratamiento, debido a la presencia de organismos patógenos (Bernal et al., 2009; Anyanwu et al., 2013; Bazrafshan et al., 2016; Jara-Samaniego et al., 2017).

Como alternativa de manejo de los residuos orgánicos está el compostaje, que es un proceso biodegradativo, aerobio y biológico, donde se transforman los residuos orgánicos en sustancias humificadas, es promovido principalmente por bacterias y hongos, cuya acción metabólica conduce a la mineralización y humificación parcial de los residuos orgánicos (Cooperband, 2002; Boulter et al., 2002; Guardabassi et al., 2003; Partanen et al., 2010; Guzmán et al., 2014); Guzmán et al., 2015) para convertirlos en productos potencialmente útiles para su aplicación en las áreas de producción de interés agroalimentario, hay suficiente evidencia que el compost es una alternativa sostenible para restaurar la fertilidad de los suelos agrícolas degradados (Bellino et al., 2014; Peigne y Girardin, 2004; Ahmad et al., 2007; De Gannes et al., 2012).

El sustrato obtenido del proceso de compostaje puede suministrar todos los macros y micro nutrientes necesarios para la obtener altos rendimientos en cultivos de interés agroalimentario (Borken et al., 2002; Ahmed et al., 2007; Anyanwu et al., 2013), por lo que puede ser utilizado como abono, en enmiendas, como acondicionador del suelo y en la recuperación de suelos erosionados (Cooperband 2002; Garrido et al., 2002; Zorpas et al., 2003; Anwar et al., 2015), obteniéndose beneficios directos como el aumento de la productividad agrícola, la mejora de la biodiversidad del suelo y la reducción de los problemas ambientales y económicos asociados con los métodos tradicionales para eliminar los residuos (Guardabassi et al., 2003; Singh y Nain, 2014).

La tasa de degradación y la calidad del compost se pueden mejorar mediante la inoculación de microorganismos. Sin embargo, los microorganismos que se utilicen para este propósito deben ser los apropiados para las características de la materia prima (Vargas-García et al., 2007 y Fan et al., 2017).

Por lo expuesto, el objetivo del presente trabajo fue evaluar el efecto de la aplicación de un inóculo nativo sobre los indicadores físicos, químicos y biológicos del compost, durante el proceso de compostaje.

\section{Materiales y métodos}

\section{Formación de pilas}

Las materias primas utilizadas en este estudio fueron las siguientes: porquinaza (estiércol de cerdos mezclada con cascarilla de arroz, y cáscara de maní. La mezcla de los residuos fue sometida a un proceso de compostaje durante 120 días, empleando el método de apilamiento por volteos. La mezcla de residuos $\left(0.33 \mathrm{~m}^{3}\right)$ se colocó en cilindros de malla metálica $(50 \mathrm{~cm}$ de altura y 96 $\mathrm{cm}$ de diámetro). El inóculo contenía un cultivo mixto de hongos (Trichoderma harzianum y Trichoderma longibrachatium) y una cepa bacteriana (Bacillus spp), perteneciente a la colección de cepas del Laboratorio de Biología Molecular de la Escuela Superior Politécnica Agropecuaria de Manabí. Se aplicaron los siguientes tratamientos expresados en $\mathrm{mL}$ del cultivo de inóculo/ $\mathrm{m}^{3}$ de mezcla de residuo: $200 \mathrm{~mL} / \mathrm{m}^{3}$ (T1); $300 \mathrm{~mL} / \mathrm{m}^{3}$ (T2); $400 \mathrm{~mL} / \mathrm{m}^{3}$ (T3) y $0 \mathrm{~mL} / \mathrm{m}^{3}$ (testigo).

\section{Análisis fisicoquímico}

La temperatura, el $\mathrm{pH}$ y la humedad se monitorearon y registraron periódicamente, la temperatura se evaluó con un termómetro de punzón en tres puntos equidistantes de la pila. Para el contenido de humedad, las muestras se secaron en estufa (Memmert) a $105^{\circ} \mathrm{C}$ durante $24 \mathrm{~h}$ (AOAC, 1990). El pH y la conductividad eléctrica de las muestras de compost se analizaron en extractos de agua $(1: 10, \mathrm{p} / \mathrm{v})$, para su determinación se utilizó un medidor de $\mathrm{pH}$ (Oakton $\mathrm{pH}$ 700) y un electrodo de conductividad (Oakton CON 700) (GómezBrandón et al., 2008). El contenido de materia orgánica se determinó por el Método volumétrico y el contenido de ceniza se midió por el método INEN 467. La relación $\mathrm{C} / \mathrm{N}$ se determinó a los 30 y a los 120 días; mientras que la concentración de N, P, K Ca, Mg, S, B, Zn, Cu y Fe se determinó a los 120 días.

\section{Análisis microbiológico}

Para el análisis microbiano se cuantificaron los siguientes grupos microbianos: (i) Coliformes totales (Método INEN 1529-8), (ii) Escherichia coli, (Método INEN 1529-8).

\section{Análisis estadístico}

El experimento se lo realizó por triplicado y los resultados se analizaron mediante el programa estadístico infostat (versión 2018). Se realizó el análisis de varianza (ANOVA), para comparar las medias de tratamiento (Di Rienzo et al., 2014). 


\section{Resultados y discusión}

\section{Evolución de la temperatura.}

La temperatura de las pilas de compostaje fue mayor a $45^{\circ} \mathrm{C}$ después de 24 horas de iniciado el proceso de compostaje en todos los tratamientos (Gráfico 1), similar a lo observado por Nutongkaew et al. (2014). Sin embargo, no se alcanzó el rango óptimo señalado por Nicholson et al. (2005) y Fourti et al. (2008), quienes mencionan que la temperatura no debe ser menor a $55^{\circ} \mathrm{C}$ durante tres días consecutivos para que ocurra la inactivación de los patógenos que están presentes en las mezclas de residuos del compostaje. La dosis de $300 \mathrm{~mL}$ obtuvo el mayor promedio de temperatura con $48.7^{\circ} \mathrm{C}$. Las temperaturas elevadas son importantes durante la fase termófila ya que aceleran la descomposición de las proteínas, grasas e hidratos de carbono complejos (Fourti et al., 2008).

\section{Evolución del pH.}

A las 24 horas, el tratamiento 2 obtuvo el máximo promedio de $\mathrm{pH}$ con 7,30 (Gráfico 2). Fourti et al. (2011) mencionan que el rango ideal de $\mathrm{pH}$ debe estar entre 6-8 para permitir una mayor tasa de descomposición, si el $\mathrm{pH}$ está fuera de este rango, afectará la actividad microbiana, prolongando los tiempos de descomposición. Los valores de $\mathrm{pH}$ al final del compostaje para los tratamientos T1, T2, T3 y T4 fueron $6.94 ; 7.00 ; 7.12$ y 6.98 respectivamente, similar a lo observado por Bazrafshan et al. (2006) y Sarika et al. (2014).

\section{Evolución del contenido de cenizas.}

El contenido de cenizas en los cuatro tratamientos varió gradualmente durante el proceso de compostaje. Los resultados muestran que el contenido de cenizas fue similar en todos los tratamientos hasta los 90

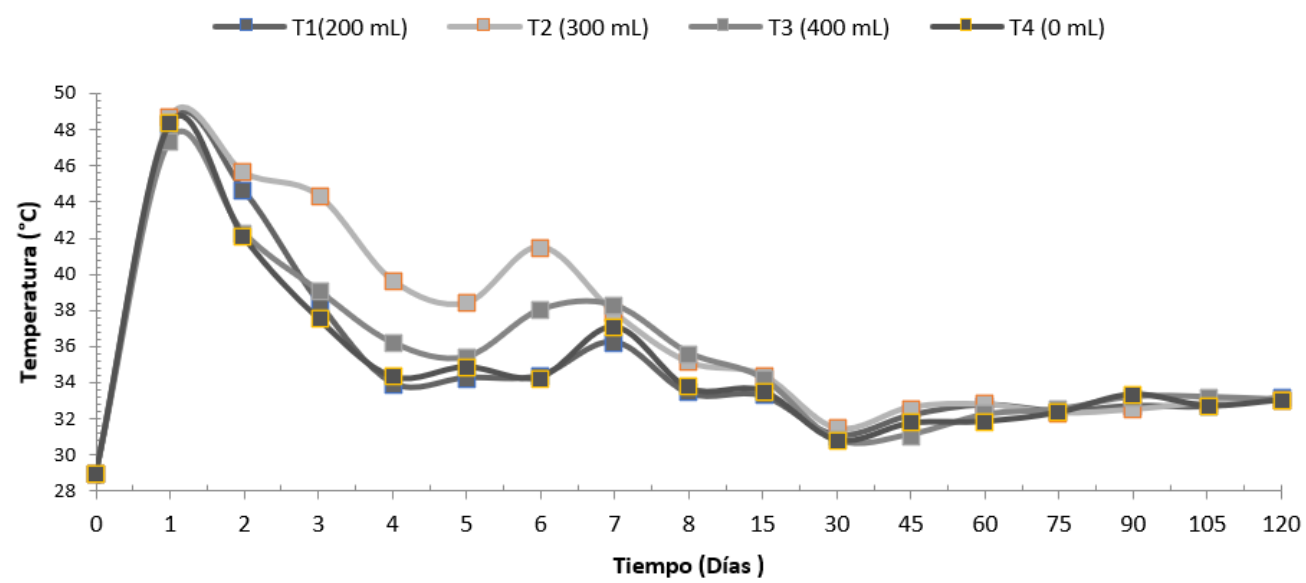

Gráfico 1. Evolución de la temperatura durante el proceso de compostaje

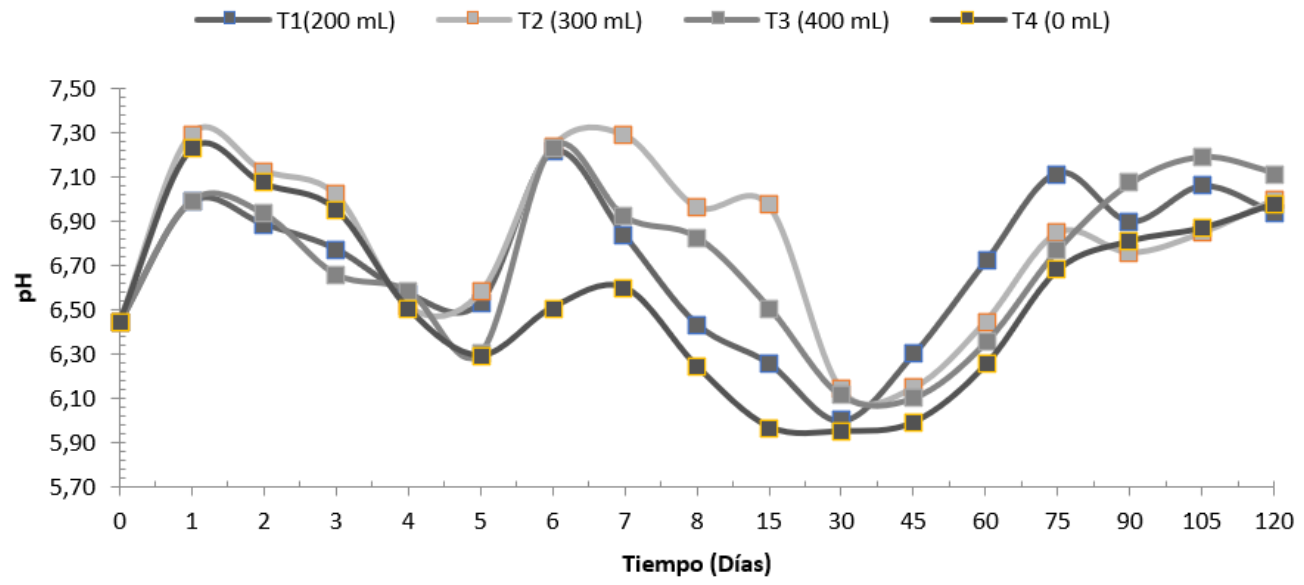

Gráfico 2. Evolución del pH durante el proceso de compostaje 
días del compostaje. A los 120 días del proceso se presentaron diferencias estadísticas entre las variantes, estableciéndose dos categorías; en el segundo rango se ubicó el T1 con $52.1 \%$ de cenizas. El resto de variantes compartieron la primera categoría estadística, con valores entre 55.9 y $56.5 \%$ de cenizas (Cuadro 1).

\section{Cuadro 1. Contenido de cenizas}

\begin{tabular}{ccccc}
\hline \multirow{2}{*}{ Dosis de inoculo } & \multicolumn{4}{c}{ Tiempo (Días) } \\
\cline { 2 - 5 } & 30 & 60 & 90 & 120 \\
\hline $200 \mathrm{~mL} / \mathrm{m}^{3}$ & $53.1^{\mathrm{a}}$ & $54.3^{\mathrm{a}}$ & $53.5^{\mathrm{a}}$ & $52.1 \mathrm{~b}$ \\
$300 \mathrm{~mL} / \mathrm{m}^{3}$ & $54.8^{\mathrm{a}}$ & $55.0^{\mathrm{a}}$ & $51.2^{\mathrm{a}}$ & $56.5 \mathrm{a}$ \\
$400 \mathrm{~mL} / \mathrm{m}^{3}$ & $54.8^{\mathrm{a}}$ & $54.6^{\mathrm{a}}$ & $52.2^{\mathrm{a}}$ & $55.9 \mathrm{a}$ \\
$0 \mathrm{~mL} / \mathrm{m}^{3}$ & $56.7^{\mathrm{a}}$ & $57.2^{\mathrm{a}}$ & $53.7^{\mathrm{a}}$ & $56.0 \mathrm{a}$ \\
\hline $\mathrm{p}$-valor & 0.9554 & 0.8567 & 0.5599 & 0.0067 \\
\hline
\end{tabular}

Letras distintas en columna indican diferencias
significativas $\mathrm{p}<0.05$ (Tukey HSD)

\section{Evolución de la CE.}

A los 30 días la CE se encontraba en un rango de 900-1400 $\mu \mathrm{S} \mathrm{cm}^{-1}$, (Cuadro 2). Posteriormente (60 días) ocurrió una disminución representativa (655-807 $\mu \mathrm{S} \mathrm{cm}^{-1}$ ). En evaluaciones posteriores (90 y 120 días) la $\mathrm{CE}$ tuvo un aumento $\left(1066 \mu \mathrm{S} \mathrm{cm} \mathrm{cm}^{-1}\right)$, provocando el incremento en la concentración de nutrientes. La conductividad eléctrica final no excedió el valor límite de $3000 \mu \mathrm{S} \mathrm{cm}^{-1}$ para compostas estables, similar a lo reportado por Gao et al. (2010).

\section{Cuadro 2. Contenido de $\mathrm{CE}\left(\mu \mathrm{S} / \mathrm{cm}^{-1}\right)$}

\begin{tabular}{ccccc}
\hline \multirow{2}{*}{$\begin{array}{c}\text { Dosis de } \\
\text { inoculo }\end{array}$} & \multicolumn{4}{c}{ Tiempo (Días) } \\
\cline { 2 - 5 } & 30 & 60 & 90 & 120 \\
\hline $200 \mathrm{~mL} / \mathrm{m}^{3}$ & $982^{\mathrm{a}}$ & $655^{\mathrm{a}}$ & $739^{\mathrm{a}}$ & $893^{\mathrm{a}}$ \\
$300 \mathrm{~mL} / \mathrm{m}^{3}$ & $1112^{\mathrm{a}}$ & $761^{\mathrm{a}}$ & $822^{\mathrm{a}}$ & $1024^{\mathrm{a}}$ \\
$400 \mathrm{~mL} / \mathrm{m}^{3}$ & $1092^{\mathrm{a}}$ & $754^{\mathrm{a}}$ & $909^{\mathrm{a}}$ & $1066^{\mathrm{a}}$ \\
$0 \mathrm{~mL} / \mathrm{m}^{3}$ & $1362^{\mathrm{a}}$ & $807^{\mathrm{a}}$ & $909^{\mathrm{a}}$ & $1034^{\mathrm{a}}$ \\
\hline $\mathrm{p}$-valor & 0.3146 & 0.1466 & 0.4472 & 0.3193 \\
\hline
\end{tabular}

Letras distintas en columna indican diferencias
significativas $\mathrm{p}<0.05$ (Tukey HSD)

\section{Evolución de la relación carbono/nitrógeno.}

La relación carbono nitrógeno $(\mathrm{C} / \mathrm{N})$ a los 120 días de compostaje disminuyó en todos los tratamientos, similar a lo reportado por Adediran et al. (2003); Baharuddin et al. (2009); Patel et al. (2015) y Halet et al. (2016). siendo el tratamiento 3 el que presentó la mayor reducción de la relación $\mathrm{C} / \mathrm{N}$ de 23 a 16 (Cuadro $3)$. Esta reducción puede atribuírsela a la pérdida de carbono y al incremento en el contenido de nitrógeno (Patel et al., 2015; Bazrafshan et al., 2016).
Cuadro 3. Evolución de la relación carbono/nitrógeno

\begin{tabular}{clc}
\hline \multirow{2}{*}{ Dosis de inoculo } & \multicolumn{2}{c}{ Tiempo (Días) } \\
\cline { 2 - 3 } & 30 & 120 \\
\hline $200 \mathrm{~mL} / \mathrm{m}^{3}$ & 30 & 29 \\
$300 \mathrm{~mL} / \mathrm{m}^{3}$ & 25 & 22 \\
$400 \mathrm{~mL} / \mathrm{m}^{3}$ & 23 & 16 \\
$0 \mathrm{~mL} / \mathrm{m}^{3}$ & 30 & 27 \\
\hline
\end{tabular}

\section{Composición química del compost.}

A los 120 días del compostaje, la concentración de macro y micro elementos de los sustratos, en todos los tratamientos, presentaron valores muy similares; lo cual, responde a las cualidades del material de partida y no a la incorporación del inóculo. Aunque los valores son bajos, hay importantes trazas de elementos nutritivos como fuente de nutriente para las plantas (Cuadro 4). Se encontró que el contenido de N\% de los compost a los 120 días para todos los tratamientos estaba entre $1.1-1.5 \%$, el valor más alto corresponde a las variantes con mayor inóculo en la pila, similar a lo reportado por Dadi, et al. (2012).

\section{Bacterias patógenas durante el compostaje.}

A pesar de que en la etapa termófila la temperatura máxima no superó los $50^{\circ} \mathrm{C}$, la densidad microbiana no deseable en el compost se redujo durante el proceso de compostaje (Gráfico 3). Sin embargo, no se logró eliminar completamente los Coliformes totales y E. coli en el producto final, similar a lo reportado por Choy et al. (2015) y Hoffmeister et al. (2018). Los valores obtenidos a los 120 días muestran que el compost que recibió la dosis de $400 \mathrm{~mL} / \mathrm{m}^{3}$ se encuentran dentro de los límites permisibles establecidos por la EPA (1994), la cual clasifica en clase A, los compostajes que finalizan con un estándar de $<1000 \mathrm{NMP} / \mathrm{g}$. de Coliformes fecales.

El análisis inicial para E. coli, en todos los tratamientos, indican que las muestras del sustrato estaban por encima de los límites establecidos en las normas para compost. Durante el proceso de compostaje la densidad de E. coli en el compost se redujo (Gráfico 4), siendo la dosis de inoculación de $400 \mathrm{~mL} / \mathrm{m}^{3}$ la que presentó el compost con menor carga patógena a los 120 días. La reducción de coliformes totales y E. coli durante el proceso de compostaje puede atribuírselo a las cepas fúngicas (T. harzianum y $T$. longibrachiatum), que compiten interespecíficamente eliminando gérmenes patógenos, al producir metabolitos secundarios volátiles y no volátiles (Monte y Llobelf, 2003). 
Cuadro 4. Características químicas del compost a los 120 días del compostaje

\begin{tabular}{|c|c|c|c|c|}
\hline \multirow{2}{*}{ Elementos } & \multicolumn{4}{|c|}{ Dosis de inoculo } \\
\hline & $200 \mathrm{~mL} / \mathrm{m}^{3}$ & $300 \mathrm{~mL} / \mathrm{m}^{3}$ & $400 \mathrm{~mL} / \mathrm{m}^{3}$ & $0 \mathrm{~mL} / \mathrm{m}^{3}$ \\
\hline Nitrógeno (\%) & 1.3 & 1.5 & 1.5 & 1.1 \\
\hline Fósforo & 0.70 & 0.62 & 0.78 & 0.80 \\
\hline Potasio & 0.90 & 0.65 & 0.79 & 0.78 \\
\hline Calcio & 1.97 & 1.54 & 1.85 & 1.65 \\
\hline Magnesio $\quad(\%)$ & 0.53 & 0.44 & 0.67 & 0.54 \\
\hline Azufre & 0.06 & 0.04 & 0.06 & 0.06 \\
\hline (ppm) & 23.67 & 20.33 & 28.33 & 25.00 \\
\hline (ppm) & 68.67 & 62.33 & 69.00 & 71.33 \\
\hline (ppm) & 29.33 & 25.67 & 31.33 & 33.00 \\
\hline (ppm) & 598.33 & 595.00 & 597.33 & 600.67 \\
\hline Manganeso (ppm) & 284.66 & 269.00 & 292.33 & 301.00 \\
\hline
\end{tabular}

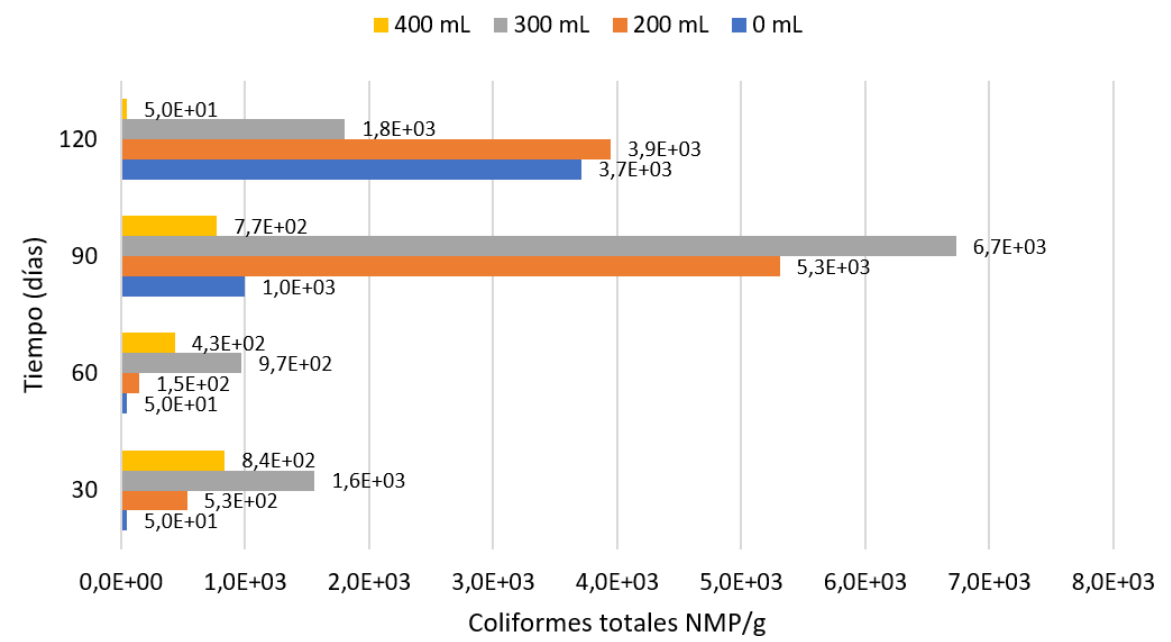

Gráfico 3. Variaciones de coliformes totales durante el proceso de compostaje

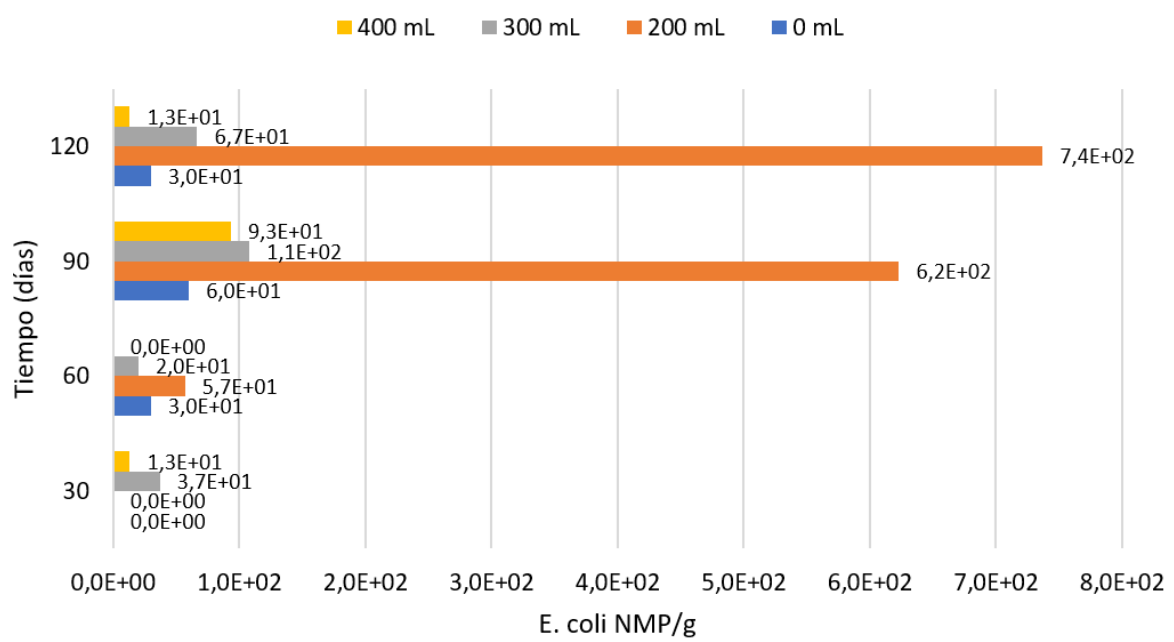

Gráfico 4. Variaciones de $E$. coli durante el proceso de compostaje 


\section{Conclusiones}

Ta aplicación del inoculo microbiano, T. harzianum, UT. longibrachiatium y Bacillus spp $\left(400 \mathrm{~mL} / \mathrm{m}^{3}\right)$ en mezclas de residuos (estiércol de cerdos mezclada con cascarilla de arroz y cáscara de maní) favoreció el proceso de compostaje para obtener un compost con mayor estabilidad y madurez de acuerdo a los parámetros físico-químicos, microbiológicos y de fitotoxicidad evaluados.

\section{Agradecimiento}

la Escuela Superior Politécnica Agropecuaria de AManabí Manuel Félix López por el auspicio y financiamiento de la investigación.

\section{Literatura citada}

Adediran, J. A., Taiwo, L. B. \& Sobulo, R. A. 2003. Effect of Organic Wastes and Method of Composting on Compost Maturity, Nutrient Composition of Compost and Yields of Two Vegetable Crops. Journal of Sustainable Agriculture, 22(4), 95-109. https://doi.org/10.1300/ J064v22n04 08 .

Ahmad, R., Jilani, G., Arshad, M., Zahir, Z. A. \& Khalid, A. 2007. Bio-conversion of organic wastes for their recycling in agriculture: an overview of perspectives and prospects. Annals of Microbiology, 57(4), 471-479. https://doi.org/10.1007/BF03175343

Ahmed, M., Idris, A. \& Omar, S. R. S. 2007. Physicochemical characterization of compost of the industrial tannery Sludge. Journal of Engineering Science and Technology, 2(1), 81-94.

Anwar, Z., Irshad, M., Fareed, I. \& Saleem, A. 2015. Characterization and Recycling of Organic Waste after Co-Composting - A Review. Journal of Agricultural Science, 7(4), 68-79. https://doi.org/10.5539/jas. v7n4p68.

Anyanwu, Ch. F., Ngohayon, S. L., Ildefonso, R. L. \& Ngohayon, J. L. 2013. Application of Indigenous Microorganisms (IMO) for Bio-Conversion of Agricultural Waste, 4(5), 7.

AOAC (Association of Official Analytical Chemists). 1990. Official Method of Analysis of the Association of Official Analytical Chemists, 15th edn. The Association of Official Analytical Chemists. U.S.A.

Baharuddin, A., Wakisaka, M., Shirai, Y., Abd. Aziz, S., Abdul Rahman, N. \& Hassan, M. A. 2009. Co-composting of empty fruit bunches and partially treated palm oil mill effluents in pilot scale. International Journal of Agricultural Research, 4(2), 69-78.

Bazrafshan, E., Zazouli, M., Bazrafshan, J. \& Bandpei, A. (2006). Evaluation of microbiological and chemical parameters during wastewater Sludge and Sawdust Co-composting. Journal of Applied Sciences and Environmental Management, 10(2). http://doi:10.4314/ jasem.v10i2.43683.

Bazrafshan, E., A. Zarei, F. Kord, N. Poormollae, S. Mahmoodi. \& M. Zazouli. 2016. Maturity and Stability Evaluation of Composted Municipal Solid Wastes. Health Scope. 5(1): 1-9.

Bellino, A., Baldantoni, D., De Nicola, F., Iovieno, P., Zaccardelli, M. \& Alfani, A. 2014. Compost amendments in agricultural ecosystems: Confirmatory path analysis to clarify the effects on soil chemical and biological properties. The Journal of Agricultural Science, 153, 1-14. https://doi.org/10.1017/S0021859614000033

Bernal, M. P., Alburquerque, J. A. \& Moral, R. 2009. Composting of animal manures and chemical criteria for compost maturity assessment. A review. Bioresource Technology, 100(22), 5444-5453. https://doi. org/10.1016/j.biortech.2008.11.027

Borken, W., Muhs, A. \& Beese, F. 2002. Application of compost in spruce forests: effects on soil respiration, basal respiration and microbial biomass. Forest Ecology and Management, 159(1-2), 49-58. https://doi. org/10.1016/S0378-1127 (01)00709-5

Boulter, J., J. Trevors, G. \& Boland, 2002. Microbial studies of compost: bacterial identification, and their potential for turfgrass patogen suppression. World Journal of Microbiology and Biotechnology. 18(7): 661-671.

Choy, SY, Wang, K., Qi, W., Wang, B., Chen, C.-L. \& Wang, J.-Y. 2015. Co-compostaje de residuos hortícolas con cáscaras de frutas, residuos de alimentos y residuos de soja. Tecnología ambiental, 36 (11), 1448-1456. doi: 10.1080 / 09593330.2014 .993728$.

Cooperband, L. 2002. The Art and Science of Composting: A resource for farmers and compost producers. University of Wisconsin-Madison, Center for Integrated Agricultural Systems.

Dadi, D. Sulaiman, H. \& Leta, S. 2012. Evaluation of Composting and the Quality of Compost from the Source Separated Municipal Solid Waste. J. Appl. Sci. Environ. Manage. 16(1): 5-10.

De Gannes, V., Eudoxie, G., Dyer, D. H. \& Hickey, W. J. 2012. Diversity and abundance of ammonia oxidizing archaea in tropical compost systems. Frontiers in Microbiology, 3. https://doi.org/10.3389/fmicb.2012.00244.

Di Rienzo J.A., Casanoves F., Balzarini M.G., Gonzalez L., Tablada M. \& Robledo C.W. 2014. InfoStat versión. Grupo InfoStat, FCA, Universidad Nacional de Córdoba, Argentina. URL http://www.infostat.com.ar.

EPA (Environmental Protection Agency) 1994. A plain English guide to the EPA part 503 biosolids rule. Office of Wastewater Management, Environmental Protection Agency, Washington, D.C. USA.

Fan Y.V., Lee C.T., Ho C.S., Klemeš J.J., Wahab R.A., Chua 
L.S. \& Sarmidi M.R., 2017, Evaluation of microbial inoculation technology for composting, Chemical Engineering Transactions, 56, 433-438 DOI:10.3303/ CET1756073

Fourti, O., Jedidi, N. \& Hassen, A. 2008. Behaviour of Main Microbiological Parameters And of Enteric Microorganisms During the Composting of Municipal Solid Wastes and Sewage Sludge in A Semi-Industrial Composting Plant. American Journal of Environmental Sciences, 4(2), 103-110. https://doi.org/10.3844/ ajessp.2008.103.110.

Fourti, O., Jedidi, N. \& Hassen, A. 2011. Comparison of methods for evaluating stability and maturity of cocomposting of municipal solid wastes and sewage sludge in semi-arid pedo-climatic condition. Natural Science, 03(02), 124-135. https://doi.org/10.4236/ns.2011.32018.

Gao, M., Liang, F., Yu, A., Li, B. \& Yang, L. 2010. Evaluation of stability and maturity during forced-aeration composting of chicken manure and sawdust at different $\mathrm{C} / \mathrm{N}$ ratios. Chemosphere, 78(5), 614-619. https://doi. org/10.1016/j.chemosphere.2009.10.056

Garrido, S., Vilchis Juárez, J., André Ramonet, C., García López, J., Alvarez Rios, A. \& Gorostieta Uribe, E. 2002. Aerobic thermophilic composting of waste sludge from gelatin-grenetine industry. Resources, Conservation and Recycling, 34, 161-173. https://doi.org/10.1016/S09213449(01)00099-4.

Gómez-Brandón, M. Lazcano, C. \& Domínguez, J. 2008. The evaluation of stability and maturity during the composting of cattle manure. Chemosphere 70: 436-444. http://doi:10.1016/j.chemosphere.2007.06.065.

Guardabassi, L., Dalsgaard A. y Sobsey M. 2003. Occurrence and survival of viruses in composted human faeces, Technical Report 32, Danish Environmental Protection Agency, Koebenhavn, Denmark. 59.

Guzmán, A., Zambrano, D., Rondón, A., Laurencio, M., Pérez, M., León, R. \& Rivera, R. (2014). Aislamiento, selección y caracterización de hongos celulíticos a partir de muestras de suelo en Manabí-Ecuador. Revista. Fac. Cienc. Agrar., Univ. 46 (2), 177-189.

Guzmán, A., Zambrano, D., Rivera, R., Rondón, A., Laurencio, M., Pérez, M. \& León, R. (2015). Aislamiento y selección de bacterias autóctonas de Manabí-Ecuador con actividad celulolítica. Cultivos Tropicales, 36 (1), 7-16.

Halet, D., Boon, N. \& Verstraete, W. 2016. Community dynamics of methanotrophic bacteria during composting of organic matter. Journal of Bioscience and Bioengineering, 101(4), 297-302. https://doi. org/10.1263/jbb.101.297.

Hoffmeister, D., Germani, J. C. \& Sand, S. T. V. D. 2018. Characterization of bacterial population during composting of municipal solid waste. Acta Scientiae Veterinariae, 33(3), 283-290.
Jara-Samaniego, J., Pérez-Murcia, M. D., Bustamante, M. A., Paredes, C., Pérez-Espinosa, A., Gavilanes-Terán, I. \& Moral, R. 2017. Development of organic fertilizers from food market waste and urban gardening by composting in Ecuador. PLOS ONE, 12(7), 1-17. https://doi. org/10.1371/journal.pone.0181621.

Monte, E. \& A. Llobell. 2003. Trichoderma in organic agriculture. p. 725-733. In V World Avocado Congress, Málaga, Spain. 19-24 October.

Nicholson, F., Groves, S. \& Chambers B., 2005. Pathogen survival during livestock manure storage and following land application. Bioresour Technol. 96(2): 135-43.

Nutongkaew, T., Duangsuwan, W., Prasertsan, S. \& Prasertsan, P. 2014. Effect of inoculum size on production of compost and enzymes from palm oil mill biogas sludge mixed with shredded palm empty fruit bunches and decanter cake. Songklanakarin J. Sci. Technol., 36(3), 275-281.

Partanen, P., Hultman, J., Paulin, L., Auvinen, P. \& Romantschuk, M. 2010. Bacterial diversity at different stages of the composting process. BMC Microbiology, 10(94), 1-11. https://doi.org/10.1186/1471-2180-10-94.

Patel, J., Yossa, I., Macarisin, D. \& Millner, P. 2015. Physical Covering for Control of Escherichia coli 0157:H7 and Salmonella spp. in Static and Windrow Composting Processes. Applied and environmental microbiology, 81(6), 2063-2074. https://doi.org/10.1128/AEM.0400214.

Peigne, J. \& Girardin, P. 2004. Environmental Impacts of Farm-Scale Composting Practices. Water Air and Soil Pollution, 153, 45-68. https://doi.org/10.1023/ B:WATE.0000019932.04020.b6.

Sabiiti, E. 2011. Utilising agricultural waste to enhance food security and conserve the environment. African Journal of Food, Agriculture, Nutrition and Development 11(6).

Sarika, D., Singh, J., Prasad, R., Vishan, I., Varma, V. S. \& Kalamdhad, A. S. 2014. Study of physico-chemical and biochemical parameters during rotary drum composting of water hyacinth. International Journal of Recycling of Organic Waste in Agriculture, 3(3). doi:10.1007/s40093014-0063-1.

Singh, S. \& Nain, L. 2014. Microorganisms in the Conversion of Agricultural Wastes to Compost. Proceedings of the Indian National Science Academy, 80(2), 473-481. https://doi.org/10.16943/ptinsa/2014/v80i2/4.

Vargas-García, M. C., Suárez-Estrella, F., López, M. J. \& Moreno, J. 2007. Effect of inoculation in composting processes: Modifications in lignocellulosic fraction. Waste Management, 27(9), 1099-1107. doi:10.1016/j. wasman.2006.06.013

Zorpas, A. A., Arapoglou, D. \& Panagiotis, K. 2003. Waste paper and clinoptilolite as a bulking material with dewatered anaerobically stabilized primary sewage sludge (DASPSS) for compost production. Waste Management, 23(1), 2735. https://doi.org/10.1016/S0956-053X(02)00042-9. 